\title{
Unique Alpha-Spectrin Mutant in a Kindred with Common Hereditary Elliptocytosis
}

Peter A. Lane," Roberta L. Shew," Terri A. larocci," Narla Mohandas, ${ }^{*}$ Taru Hays, and William C. Mentzer*;

Departments of ${ }^{*}$ Pediatrics and ${ }^{\ddagger}$ Laboratory Medicine, University of California, San Francisco, California $94110 ;$ and ${ }^{\S}$ Denver Children's Hospital and the Department of Pediatrics, University of Colorado School of Medicine, Denver, Colorado 80262

\begin{abstract}
We report here a unique variant of alpha spectrin in a kindred with hereditary elliptocytosis. This novel red blood cell-membrane protein migrated to a position between the normal alphaand beta-spectrin subunits in SDS polyacrylamide gel electrophoresis. It was identified as an alpha spectrin by its binding to anti-alpha spectrin antibodies, by the absence of a phosphorylation site, and by the normal 1:1 stoichiometry between total alpha- and beta-spectrin molecules. The quantity of the alphaspectrin mutant, expressed as a percentage of the total alpha spectrin, varied from 9.9-45.2\% among six affected individuals. Two-dimensional electrophoretic analysis of spectrin tryptic digests was qualitatively normal but showed a decreased quantity of a normal alpha IV fragment. The variable quantity of alphaspectrin mutant among family members correlated directly with the increased percentage of spectrin dimers in cold low ionic strength spectrin extracts $(r=0.92)$ and inversely with red blood cell ghost mechanical stability $(r=-0.98)$. The data suggest that this new alpha-spectrin mutant is responsible for decreased spectrin dimer-dimer association and for red cell instability in affected individuals.
\end{abstract}

\section{Introduction}

The erythrocyte membrane skeleton plays an important role in the maintenance of red blood cell $(\mathrm{RBC})^{1}$ shape, deformability, and stability (1-3). Spectrin, the principal protein of the membrane skeleton, is composed of alpha and beta subunits. These two filamentous polypeptides are aligned side by side to form spectrin heterodimers, which in turn self-associate to form spectrin tetramers and higher molecular weight oligomers. Spectrin also binds to another skeletal protein, actin, in the presence of a third protein, band 4.1, to form a richly anastamosing network adjacent to the inner aspect of the membrane lipid bilayer. The skeleton is anchored to the bilayer by yet another protein, an-

This work was presented in part to the American Society of Hematology, New Orleans, LA (1985, Blood, 66:(Suppl. 1)35a). Address reprint requests to Dr. Lane, Northern California Comprehensive Sickle Cell Center, Building 20, Room 2108, San Francisco General Hospital, 1001 Potrero Avenue, San Francisco, CA 94110. 1986.

Received for publication 24 March 1986 and in revised form 6 October

1. Abbreviations used in this paper: $\mathrm{HE}$, hereditary elliptocytosis; $\mathrm{Hgb}$, hemoglobin; PMSF, phenylmethylsulfonyl fluoride; RBC, red blood cell.

J. Clin. Invest.

(C) The American Society for Clinical Investigation, Inc.

0021-9738/87/03/0989/08 \$1.00

Volume 79, March 1987, 989-996 kyrin, which binds to both spectrin and the integral protein, band 3.

Abnormalities in the skeleton or its attachments have been discovered in a number of congenital hemolytic anemias. For example, molecular defects described in kindreds with hereditary elliptocytosis (HE) include a quantitative deficiency of protein 4.1 (4), defective ankyrin-band 3 binding (5), and a number of qualitative defects in spectrin (6-16). Most of alpha- and betaspectrin defects in HE result in diminished self-association of affected spectrin heterodimers (7-16), leading to reduced formation of higher molecular weight forms of spectrin and, thus, to skeletal instability. Several previously described defects in alpha spectrin have been distinguished from one another by differing patterns of spectrin peptides generated by limited tryptic digestion (6, 10-14). One beta-spectrin mutant exhibited defective binding to ankyrin (11), while several others displayed apparent alterations in molecular weight as estimated by SDS polyacrylamide gel electrophoresis (PAGE) $(15,16)$.

We now report a new alpha-spectrin defect in a family with HE. This mutant alpha spectrin is unique because of its anomalous migration on SDS PAGE. A marked heterogeneity in the relative quantity of the alpha-spectrin mutant was found among affected family members. The strong correlation of the quantity of the mutant polypeptide with the extent of defective dimerdimer association and with skeletal instability underscores the importance of an intact spectrin complex for normal skeletal function.

\section{Methods}

Clinical material. Nine family members spanning three generations were available for study. These individuals are indicated in the pedigree (Fig. 1), and representative hematologic values for each are listed in Table I.

The propositus, $\mathrm{III}_{2}$, a 23-mo-old boy, exhibited anemia, hyperbilirubinemia requiring phototherapy, and striking $\mathrm{RBC}$ poikilocytosis at birth. His anemia reached a nadir (hemoglobin [Hgb] $7.9 \mathrm{gm} / \mathrm{dl}$, reticulocyte count $13.7 \%$ ) at $6 \mathrm{wk}$ of age and then gradually improved without specific therapy. Simultaneously, RBC morphology evolved from poikilocytosis (Fig. $2 A$ ) to elliptocytosis (Fig. $2 B$ ) and hyperbilirubinemia disappeared. The only sibling, $\mathrm{III}_{1}$, a 4-yr-old boy who had hyperbilirubinemia at birth, now exhibits elliptocytosis without poikilocytosis. The mother, $\mathrm{II}_{2}$, of German/English/Irish descent, exhibited hyperbilirubinemia at birth and hemolytic anemia $(\mathrm{Hgb} 8.5 \mathrm{gm} / \mathrm{dl}$, reticulocytes $15 \%$ ) during her pregnancy with the propositus. Currently, her RBC morphology is that of elliptocytosis (Fig. $2 C$ ). The father, $\mathrm{II}_{1}$, of English descent, is hematologically normal (Fig. $2 D$ ). The maternal grandfather of the propositus, $I_{1}$, underwent splenectomy at age $27 \mathrm{yr}$. His ${ }^{51} \mathrm{Cr}$ RBC survival studies demonstrated a $t_{1 / 2}$ of $13 \mathrm{~d}$ (control 26-30 d) before splenectomy and a $t_{1 / 2}$ of $30 \mathrm{~d}$ post-splenectomy.

Other family members, not available for study and not included in Fig. 1, include five siblings of the maternal grandfather, four of whom have $\mathrm{HE}$. Of these four, three have undergone splenectomy, and the other has splenomegaly. Their mother, the great-grandmother of the propositus, also had elliptocytosis. 


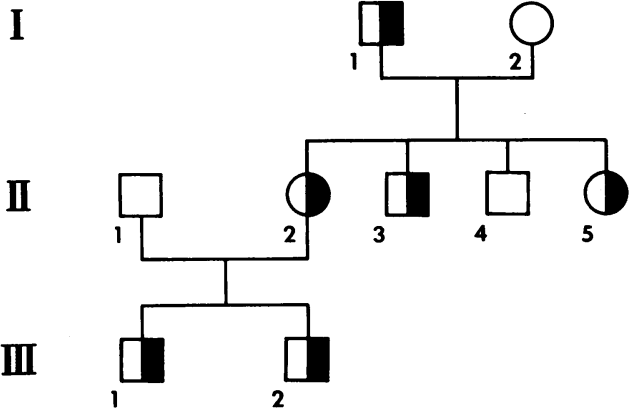

Figure 1. Pedigree of kindred with HE. Half-solid circles, individuals with elliptocytosis; open circles, individuals with normal erythrocytes. Note that $\mathrm{II}_{3}$ is depicted as having $\mathrm{HE}$ because both mutant alpha spectrin and increased spectrin dimers are present in his erythrocytes. However, his elliptocyte count is not significantly elevated.

Materials. Electrophoresis reagents were purchased from Bio-Rad Laboratories, (Richmond, CA); Tris- $\mathrm{HCl}$, phenylmethylsulfonyl fluoride (PMSF), diisopropylfluorophosphate, horseradish peroxidase-conjugated sheep anti-mouse antibodies, and 4-chloronaphthol from Sigma Chemical Co. (St. Louis, MO); and Seakem agarose from FMC Corp., Marine Colloids Div., (Rockland, ME). 2-Mercaptoethanol was purchased from Eastman Kodak Co., (Rochester, NY). ${ }^{32} \mathrm{P}\left(\mathrm{H}_{3} \mathrm{PO}_{4}\right)$ came from ICN Biomedicals Inc. (Irvine, CA). Horseradish peroxidase-conjugated goat anti-rabbit IgG antibodies were purchased from Pel-Freeze Biologicals (Rogers, AR).

Phlebotomy and routine hematologic studies. Blood samples were obtained by routine phlebotomy under a protocol authorized by the Committee on Human Experimentation, University of California (San Francisco, CA). EDTA anticoagulant was used for routine blood counts, reticulocyte determinations, and blood group phenotyping. For all other studies, samples were anticoagulated in acid citrate dextrose and stored at $4^{\circ} \mathrm{C}$ until studied. Morphologic and heat fragmentation studies were performed within $12 \mathrm{~h}$ and all other studies within $48 \mathrm{~h}$ of phlebotomy.

Morphology and RBC thermal stability. RBC morphology was determined by review of Wright-stained peripheral blood smears as well as by Nomarski interference phase-contrast microscopy of fresh RBCs fixed in $1 \%$ glutaraldehyde in phosphate-buffered saline (pH 7.4, 290 mosmol). Elliptocytes were counted by measuring the length and width of 200 fixed RBCs. Cells with a width-to-length ratio of $<0.78$ were classified as elliptocytes (17). The susceptibility of RBCs to heat-induced budding and fragmentation was assessed by incubating washed cells for $10 \mathrm{~min}$ at temperatures varying in $1^{\circ} \mathrm{C}$ increments from $45^{\circ}$ to $50^{\circ} \mathrm{C}$ (18). The lowest temperature at which $10 \%$ or more of the cells showed

Table I. Blood Counts in Family with HE

\begin{tabular}{lllll}
\hline Sample & $\mathrm{Hgb}$ & $\mathrm{MCV}$ & Reticulocytes & Elliptocytes* \\
\hline & $g / d l$ & $f l$ & $\times 10^{3} / \mathrm{mm}^{3}$ & $\%$ \\
$\mathrm{I}_{1}$ & 16.7 & 95 & 44.9 & 61.5 \\
$\mathrm{I}_{2}$ & 14.3 & 85 & 71.5 & 8.0 \\
$\mathrm{II}_{1}$ & 15.0 & 89 & 55.6 & 1.0 \\
$\mathrm{II}_{2}$ & 13.6 & 90 & 37.7 & 87.0 \\
$\mathrm{II}_{3}$ & 16.3 & 92 & 160.3 & 7.5 \\
$\mathrm{II}_{4}$ & 18.0 & 91 & - & 1.0 \\
$\mathrm{II}_{5}$ & 15.1 & 84 & 71.5 & 25.0 \\
$\mathrm{III}_{1}$ & 11.8 & 79 & 72.0 & 32.5 \\
$\mathrm{III}_{2}$ & 11.8 & 73 & 132.6 & 25.0 \\
\end{tabular}

* Normal range, $1-8 \%$ budding was determined by examining fixed cells with phase-contrast microscopy.

Stractan density gradient centrifugation. Separation of RBCs according to their buoyant density was accomplished by discontinuous stractan density gradient centrifugation as described by Clark and her co-workers (19). Five subpopulations of RBCs were harvested from each gradient and ghosts were prepared as described below.

Analysis of RBC membrane proteins. Leukocytes and platelets were removed by passing blood through columns of alpha cellulose and microcrystalline cellulose (20). RBC ghosts were then prepared at $0-4^{\circ} \mathrm{C}$ as described by Dodge et al. (21), with $0.2 \mathrm{mM}$ PMSF added to minimize proteolysis.

Fairbanks nonlinear 3.5-17\% polyacrylamide gradient SDS slab gels (22) were used for electrophoretic examination of RBC membrane proteins and to quantitate the spectrin to band 3 ratio. Ghosts or whole RBC were diluted 1:5 in SDS sample buffer (23). After electrophoresis and staining with Coomassie Blue (22), relative quantities of spectrin subunits were determined by densitometric scanning. The spectrin to band 3 ratio was determined by excising the stained bands (spectrin and band 3 ), eluting the dye in $25 \%$ pyridine overnight, and reading the absorbance at $605 \mathrm{~nm}$ (24).

For immunological analysis, RBC membrane proteins were transferred from SDS slab gels onto nitrocellulose paper (0.45-nm pores) by overnight electroelution $(25,26)$. After blocking the remaining binding sites with bovine serum albumin (BSA) the papers were incubated overnight with anti-spectrin antibody. Polyclonal rabbit anti-spectrin antibody was obtained from Joel Chasis. Polyclonal rabbit anti-beta spectrin antibody, polyclonal rabbit anti-alpha spectrin antibody (specific for the alpha I tryptic domain), and monoclonal mouse anti-alpha spectrin antibody (specific for the alpha IV domain) were provided by S. C. Liu and Jiri Palek. After antibody binding, the papers were incubated for 4 $h$ at room temperature with horseradish peroxidase-conjugated goat antirabbit or sheep anti-mouse IgG antibodies. Staining was accomplished with 4-chloronaphthol and hydrogen peroxide.

Membrane protein phosphorylation. Washed erythrocytes were incubated for $16 \mathrm{~h}$ at $37^{\circ} \mathrm{C}$ in normal saline with $50 \mathrm{mM}$ imidazole $(\mathrm{pH}$ 7.4), $31 \mathrm{mM}$ glucose, $5 \mathrm{mM}$ adenosine, $250 \mathrm{U} / \mathrm{ml}$ penicillin, $250 \mathrm{U} / \mathrm{ml}$ streptomycin and $1 \mathrm{mCi}^{32} \mathrm{P}\left(\mathrm{H}_{3} \mathrm{PO}_{4}\right)$ per cubic centimeter of packed RBC (27). After the incubation, the RBCs were washed three times in normal saline. Ghosts were prepared as above and membrane proteins electrophoresed into a Fairbanks nonlinear 3.5-17\% acrylamide gradient SDS slab gel. Autoradiographs were made by 2 -h exposure of Kodak XAR-2 X-ray film to the dried gels in an intensifying cassette.

Spectrin analysis. RBC membrane ghosts were prepared at $0-4^{\circ} \mathrm{C}$ as described above. PMSF ( $0.2 \mathrm{mM}$ final concentration) was added to all preparative solutions to prevent proteolysis. Spectrin extract was obtained from fresh ghosts by low ionic strength extraction for $16 \mathrm{~h}$ at 0 $4^{\circ} \mathrm{C}$ according to Lawler and his colleagues (28). The fraction of spectrin in the dimeric form was determined by low temperature $\left(0-4^{\circ} \mathrm{C}\right)$, nondenaturing $2.5 \%$ acrylamide, and $0.3 \%$ agarose composite gel electrophoresis (29). Spectrin dimers and tetramers were quantitated by densitometry and the percentage of spectrin in the dimeric form calculated (18). For analysis of the alpha- and beta-subunit composition of spectrin dimers and tetramers, spectrin extracts were first subjected to nondenaturing gel electrophoresis and then analyzed in the second dimension in nonlinear $4.5-17 \%$ polyacrylamide gradient Laemmli SDS slab gels (23).

Cold low ionic strength spectrin extract was also subjected to limited tryptic digestion. PMSF was removed by overnight dialysis at $4^{\circ} \mathrm{C}$ in $200 \mathrm{vol}$ of $20 \mathrm{mM}$ Tris buffer (pH 8.0) (30). The protein concentration of the extracts was determined with the Bio-Rad protein assay (Bio-Rad Laboratories) using BSA as the standard. The spectrin extracts were subjected to limited tryptic digestion for $16 \mathrm{~h}$ at $4^{\circ} \mathrm{C}$ using an enzyme to substrate ratio of $1: 100$ or 1:200 (wt/wt) (31). Digestion was terminated by adding diisopropylfluorophosphate to a $1 \mathrm{mM}$ final concentration. The spectrin tryptic fragments were analyzed by two-dimensional electrophoresis. Isoelectric focusing tube gels were prepared by the method of O'Farrell with modifications $(32,33)$. The focused proteins were elec- 

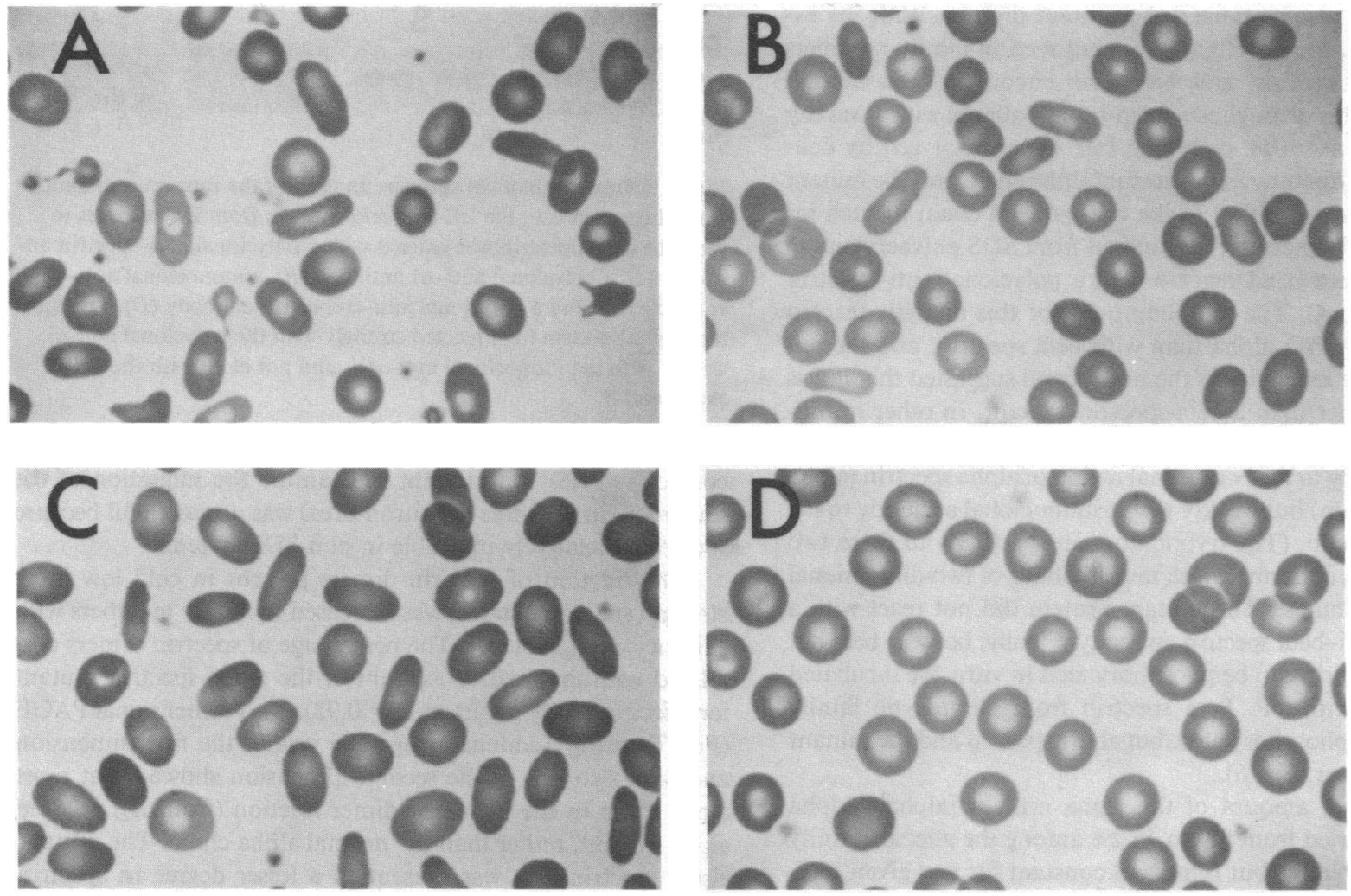

Figure 2. Photomicrographs of Wright-stained peripheral blood smears. RBC morphology in $\mathrm{III}_{2}$ at 7 wk of age showed elliptocytes and numerous micropoikilocytes with thin budlike projections $(A)$. At 17 mo of age his RBC morphology had evolved to that typical of $\mathrm{HE}(B)$. His mother, $\mathrm{II}_{2}$, also had elliptocytes $(C)$, while his father, $\mathrm{II}_{1}$, showed normal RBC morphology $(D)$.

trophoresed into the second dimension in a linear $10-15 \%$ polyacrylamide gradient SDS slab gel (23).

Erythrocyte ghost mechanical stability. RBC ghost fragmentation was assayed in a Technicon digital-processing ektacytometer (34). Resealed ghosts were suspended in a dextran solution of $97 \mathrm{cp}$ viscosity (dextran $40,000,35 \mathrm{~g} / \mathrm{dl} \mathrm{wt} / \mathrm{vol}, 290 \mathrm{mosmol} / \mathrm{kg}, \mathrm{pH} 7.4)$. These samples were subjected to a continuous shear stress of 750 dynes $/ \mathrm{cm}^{2}$, which resulted in progressive fragmentation of the intact membranes into nondeformable spherical fragments. This process was detected as a decrease in the deformability index, which was monitored as a function of time. For comparison of the fragmentation of different samples of ghosts, the time required for ghost deformability to decrease to $60 \%$ of its maximal value (the $T_{60}$ ) was determined. The ratio of the observed $T_{60}$ to that of a simultaneously run normal control sample was then used to calculate relative ghost mechanical stability expressed as a percentage of the normal value.

\section{Results}

Table I summarizes routine hematology values. Family members with elliptocytosis were not anemic, but demonstrated variable elevations in reticulocyte counts. With the exception of $\mathrm{II}_{3}$, the percentage of elliptocytes ranged from 25 to $87 \%$ in affected family members, but was $\leq 8 \%$ in family members without elliptocytosis. Blood group phenotyping of all nine family members was compatible with linkage between elliptocytosis and the Duffy (Fy) antigen, but was inconclusive due to the limited variability of phenotypes among individuals (data not shown).

We determined the minimum temperature necessary to induce erythrocyte budding to allow comparison with other spectrin defects known to cause elliptocytosis, many of which have demonstrated an increased sensitivity to heat $(6,8,11,12,15)$. Control samples and RBCs from unaffected family members exhibited budding at $49^{\circ} \mathrm{C}$. Erythrocytes from the three family members with the greatest amount of mutant spectrin $\left(\mathrm{I}_{1}, \mathrm{III}_{1}\right.$, and $\mathrm{III}_{2}$ ) sometimes showed budding at $48^{\circ} \mathrm{C}$, but at other times not below $49^{\circ} \mathrm{C}$. The RBCs of other family members with lesser amounts of the mutant were normal.

SDS PAGE of RBC ghost membrane proteins from family members with elliptocytosis revealed a novel protein that migrated between band 1, or alpha spectrin, and band 2, or beta spectrin (Fig. 3). Whole RBCs, solubilized in SDS and analyzed

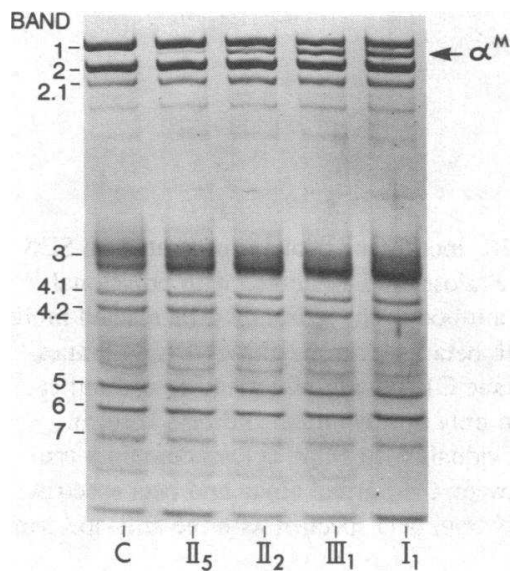

Figure 3. Erythrocyte membrane proteins separated by nonlinear $3.5-$ $17 \%$ polyacrylamide gradient Fairbanks SDS PAGE. Lane $C$ contains normal membrane proteins. The other lanes show membrane proteins from four individuals with elliptocytosis (lanes $\mathrm{II}_{5}, \mathrm{II}_{2}, \mathrm{III}_{1}$, and $\left.I_{1}\right)$. The mutant protein $\left(\alpha^{M}\right)$ is present in variable quantity at a position between band 1 (alpha spectrin) and band 2 (beta spectrin). 
on these gels, also contained the mutant protein. With the exception of $\mathrm{II}_{3}$, the novel band was not seen in family members without elliptocytosis, and was never encountered in normal control samples or in ghosts from other patients with a variety of hemolytic anemias, including HE. When analyzed by densitometry, the quantity of the normal alpha chain plus the mutant protein was equal to that of the normal beta chain in each individual. On Western blots prepared from SDS polyacrylamide gels, the mutant band reacted with a polyclonal anti-spectrin antibody (Fig. 4). The antibody used for this analysis reacted more strongly with alpha than with beta spectrin, and the relatively intense reactivity of the novel band suggested that it was an alpha- rather than a beta-spectrin mutant. In other immunoblots (Fig. 5), the mutant spectrin reacted strongly to a polyclonal antibody to the $\mathrm{N}$ terminal region of alpha spectrin (alpha I tryptic domain) but weakly with a monoclonal antibody to the alpha IV domain. (The tryptic domain specificity of these two antibodies was confirmed with immunoblots of two-dimensional tryptic digest maps.) The mutant protein did not react with a polyclonal anti-beta spectrin antibody. Finally, because beta but not alpha spectrin can be phosphorylated in vitro, we incubated erythrocytes with ${ }^{32} \mathrm{P}$. Beta spectrin from elliptocytic family members was phosphorylated, but alpha spectrin and the mutant protein were not (Fig. 6).

The relative amount of the alpha mutant [alpha ${ }^{\mathrm{M}} /($ alpha + alpha $\left.^{\mathrm{M}}\right)$ ] varied from 9.9 to $45.2 \%$ among the affected family members (Table II), but remained constant for any given individual on serial analysis over a period of $2 \mathrm{yr}$. In addition, reticulocyte-enriched RBC subpopulations (from $I_{1}$ ) showed no difference from denser erythrocytes in the relative amount of the mutant protein. The amount of total spectrin present in the RBC ghosts from affected family members, estimated by measurement of spectrin to band 3 ratios, was normal (0.97-1.06;

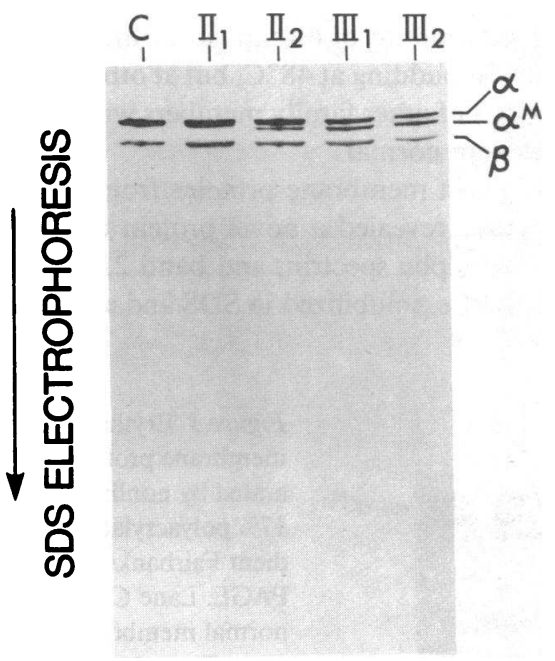

Figure 4. Immunoblot of RBC membrane proteins separated on SDS PAGE, transferred to nitrocellulose, and incubated with polyclonal rabbit anti-human spectrin antibody. The antibody used reacted more strongly with alpha than with beta spectrin. Erythrocyte membranes from an unrelated control (lane $C$ ) and a family member without elliptocytosis (lane $\mathrm{II}_{1}$ ) contain only normal alpha and beta spectrin. Erythrocytes from three individuals with elliptocytosis contain a mutant spectrin that is seen between the normal alpha and beta spectrin and that reacts more strongly than beta spectrin with the anti-spectrin antibody.

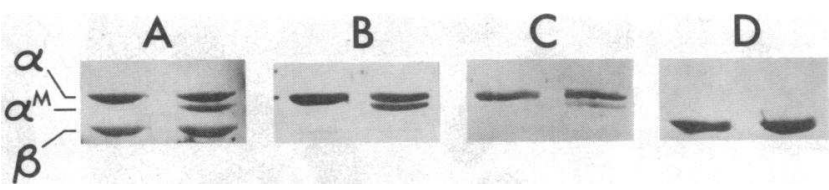

Figure 5. Immunoblots of spectrin. In each of the four immunoblots, control spectrin is in the left lane and spectrin from HE red cells in the right lane. Spectrin was reacted with a polyclonal anti-spectrin antibody $(A)$, a polyclonal anti- $\alpha \mathrm{I}$ antibody $(B)$, a monoclonal anti- $\alpha \mathrm{IV}$ antibody $(C)$, and a polyclonal anti- $\beta$ spectrin antibody $(D)$. The mutant alpha spectrin $\left(\alpha^{\mathrm{M}}\right)$ reacted strongly with the polyclonal anti- $\alpha \mathrm{I}$, weakly with the monoclonal anti- $\alpha \mathrm{IV}$, and not at all with the polyclonal anti- $\beta$.

control = 1.06). An attempt to examine the migration of the mutant in an SDS-free gel (titron-urea) was unsuccessful because spectrin is relatively insoluble in non-SDS systems.

The fraction of spectrin dimers present in cold low ionic strength spectrin extracts was increased in family members with elliptocytosis (Table II). The percentage of spectrin dimers correlated with the relative amount of the alpha-spectrin mutant for affected family members $(r=0.92)$. Two-dimensional PAGE (Fig. 7), using nondenaturing tube gels in the first dimension and SDS slab gels in the second dimension showed that most alpha chain in the increased dimer fraction (from $\mathrm{II}_{2}$ ) was the alpha mutant, rather than the normal alpha chain. The mutant alpha spectrin also was present to a lesser degree in spectrin tetramers and higher molecular weight oligomers.

Low ionic strength extracts from the three elliptocytic family members with $>40 \%$ alpha spectrin mutant were subjected to limited tryptic digestion at $4^{\circ} \mathrm{C}$ for $16 \mathrm{~h}$. The five normal alphaspectrin tryptic domains were identified by comparison with controls and published maps $(14,31)$. The only abnormality was a decrease in the smaller of two normal alpha IV fragments (Fig. 8). Immunoblots of these two-dimensional gels were prepared with the polyclonal anti-alpha I spectrin antibody and no differences between mutant and control spectrin were observed (data not shown). In addition, spectrin tryptic fragments (from $\mathrm{I}_{1}$ ) were analyzed by SDS PAGE after 1,3 , and $20 \mathrm{~h}$ of digestion and no difference from control was noted (data not shown).

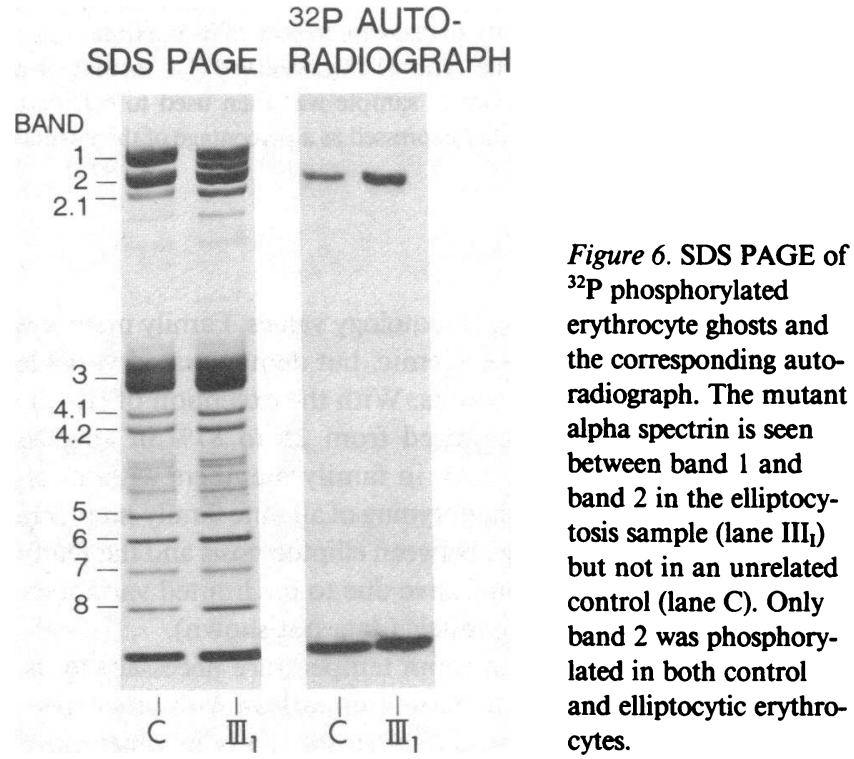


Table II. Effects of Mutant Spectrin on Spectrin

Dimers and on Ghost Mechanical Stability

\begin{tabular}{|c|c|c|c|}
\hline Sample & $\begin{array}{l}\text { Mutant spectrin } \\
\frac{\alpha^{M}}{\alpha+\alpha^{M}}\end{array}$ & $\begin{array}{l}\text { Spectrin } \\
\text { dimers* }\end{array}$ & $\begin{array}{l}\text { Ghost mechanical } \\
\text { stability }{ }^{*}\end{array}$ \\
\hline & $\%$ & $\%$ & \% of normal \\
\hline $\mathrm{III}_{2}$ & 45.2 & 19.0 & 12.0 \\
\hline III $_{1}$ & 41.7 & 15.7 & 10.6 \\
\hline$I_{1}$ & 40.1 & 20.0 & 10.3 \\
\hline $\mathrm{II}_{2}$ & 27.7 & 15.1 & 31.7 \\
\hline $\mathrm{II}_{3}$ & 10.2 & 8.4 & 89.4 \\
\hline $\mathrm{II}_{5}$ & 9.9 & 11.3 & 99.2 \\
\hline $\mathbf{I}_{2}$ & 0 & 6.2 & 111.4 \\
\hline II $_{1}$ & 0 & 3.4 & 94.5 \\
\hline $\mathrm{II}_{4}$ & 0 & 3.7 & 99.5 \\
\hline Controls & 0 & $5.4 \pm 1.6^{8}$ & $100.0 \pm 7.5^{8}$ \\
\hline
\end{tabular}

* Percentage of spectrin dimers correlated with the amount of mutant spectrin $(r=0.92)$.

₹ Ghost mechanical stability correlated inversely with the amount of mutant spectrin $(r=-0.98)$.

\pm SD.

The mechanical stability of RBC ghosts subjected to a constant shear stress in the ektacytometer was diminished in proportion to the amount of alpha-spectrin mutant present in those ghosts (Table II). The correlation coefficient between ghost me-
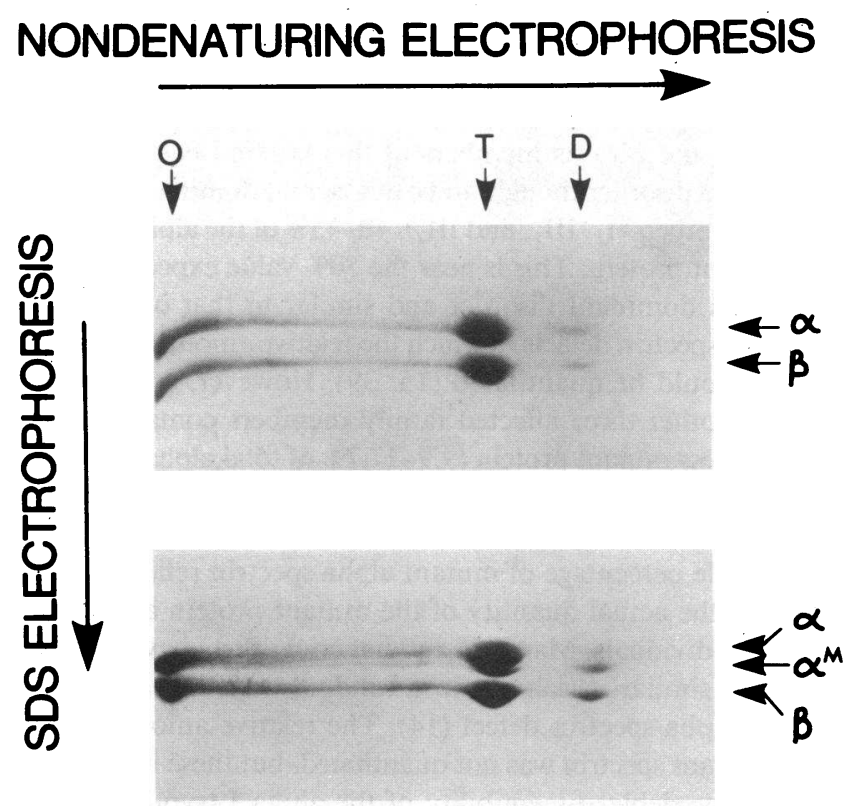

Figure 7. Two-dimensional electrophoresis of cold low ionic strength spectrin extract. In the first dimension, spectrin oligomers $(O)$ have been separated from tetramers $(T)$ and dimers $(D)$ by nondenaturing agarose PAGE. The second dimension has separated the alpha spectrin from beta spectrin. The top gel shows spectrin from a control subject. In the bottom gel, which contains spectrin from an individual with elliptocytosis, the first dimension shows an increase in the percentage of spectrin dimers to $15 \%$. The second dimension shows that most alpha spectrin in the increased dimer fraction is the alpha mutant, rather than the normal alpha chain. Note the presence of small amounts of mutant spectrin in tetramers and oligomers. chanical stability and the amount of alpha-spectrin mutant was -0.98 .

\section{Discussion}

The novel membrane protein identified in family members with elliptocytosis is an alpha-spectrin mutant because $(a)$ it reacted more strongly than beta spectrin with an anti-spectrin antibody that has greater affinity for alpha than beta spectrin, $(b)$ it reacted with a polyclonal anti-alpha spectrin antibody but not with an anti-beta spectrin antibody, (c) unlike beta spectrin, it could not be phosphorylated, and $(d)$ the amount of normal alpha spectrin was decreased by an amount equivalent to the mutant in every instance, yielding the expected 1:1 stoichiometry between alphaand beta-spectrin molecules.

To our knowledge this mutant is the first alpha spectrin to be described with anomolous migration in SDS PAGE. This finding is not an artifact of in vitro proteolysis because the quantity of the mutant protein was the same when intact RBCs were solubilized in SDS for electrophoresis, thus omitting the ghosting procedure. In addition, the quantity of the mutant protein for each individual was constant on repeated assays over a 2 -yr time span.

Strong evidence indicates that the mutant protein is directly responsible for the elliptocytosis phenotype observed in this family. With the exception of $\mathrm{II}_{3}$, only family members with elliptocytosis were found to have the mutant. In addition, the quantity of the mutant protein correlated well with other indicators of skeletal dysfunction. RBCs from these individuals, like many others with elliptocytosis, contained an increased percentage of spectrin dimers in cold low ionic strength spectrin extracts (7-16), indicating a decreased ability of spectrin dimers to self-associate and form spectrin tetramers and higher molecular weight oligomers. The direct role of the mutant alpha spectrin in defective dimer-dimer association was demonstrated by showing that the mutant, rather than the normal alpha chain, predominated in the increased dimer fraction. The role of the mutant alpha spectrin in defective dimer self-association was also supported by analysis of the variability among family members. Those with greater amounts of the mutant protein were those with a greater percentage of spectrin in the dimeric form.

In other kindreds with $\mathrm{HE}$, impaired spectrin dimer-dimer association has been linked with membrane skeletal instability $(7,9,34)$. We measured skeletal stability in the ektacytometer as a function of $\mathrm{RBC}$ ghost fragmentation. RBCs from individuals with greater amounts of the mutant protein demonstrated more rapid ghost fragmentation than individuals with less mutant. Thus, in this family, defective self-association of the (alpha ${ }^{\mathrm{M}}$-beta) dimer results in membrane skeletal instability. Presumably, this is responsible for the hemolysis observed in these individuals.

Palek has separated common HE into nine subtypes (35). The apparent autosomal dominant inheritance, RBC morphology, and slight reticulocytosis observed in this family best fit the subtype of common HE with minimal hemolysis. However, the propositus had more severe hemolysis and poikilocytosis in infancy, thus fitting the subtype, common HE with infantile poikilocytosis. The history of significant neonatal jaundice in his brother and their mother suggests that they also may have passed through a period of infantile poikilocytosis, but this cannot be confirmed in retrospect. None of the affected individuals is currently anemic, and there was a poor correlation between the 


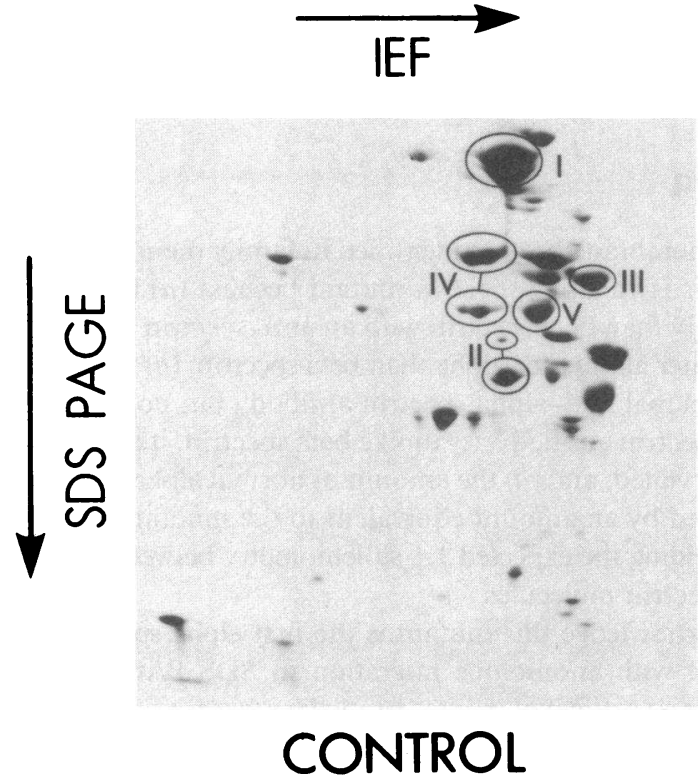

Figure 8. Two-dimensional electrophoretic analysis of spectrin extracts after limited tryptic digestion. In the first dimension, $130 \mu \mathrm{g}$ of tryptic fragments were focused across a pH gradient of 3.5-10. The second dimension separation is by linear Laemmli SDS PAGE (10-15\% acryl-

percentage of mutant alpha spectrin and the number of elliptocytes in the peripheral blood $(r=0.63)$.

The precise molecular defect remains to be defined. Anomalous migration in SDS PAGE suggests a shortening of the alphaspectrin polypeptide. Alternatively, amino acid substitutions that alter the hydrophobicity of a protein may affect mobility in SDS PAGE by altering SDS binding to the protein (36-38). Limited tryptic digestion of low ionic strength spectrin extracts containing 40-45\% mutant spectrin did not show any abnormal peptides. One possible explanation for this finding is that the site of shortening or mutation is a region of the spectrin molecule normally lost during the overnight tryptic digestion procedure. In an attempt to address this possibility, we analyzed spectrin tryptic fragments after only 1 and $3 \mathrm{~h}$ of digestion and no differences from control were noted in these less digested samples. Another possibility is that an abnormal tryptic peptide has gone undetected because of co-migration with a normal fragment. Because other alpha-spectrin mutants associated with increased spectrin dimers have demonstrated abnormalities in the $80-\mathrm{kD}$ alpha I tryptic domain $(10,12-14,28)$, we used a polyclonal anti-alpha I antibody to search for any novel alpha I tryptic fragments that might have escaped recognition on Coomassie-stained gels. Immunoblots of two-dimensional tryptic peptide maps showed that this antibody reacted normally with the 80 - and 74-kD fragments generated from digestion of patient spectrin, and no abnormal alpha I fragments were detected.

Two findings from this study suggest the possibility of an alpha IV spectrin abnormality. The first is the decrease in the smaller alpha IV fragment noted in Coomassie-stained gels. The second finding is the decreased reactivity of the undigested mutant protein with a monoclonal antibody to the alpha IV domain. It is difficult to draw a firm conclusion from these observations. The tryptic digest abnormality may be real or merely an artifact due to subtle differences between samples in the extent of digestion. The decreased affinity of the undigested mutant protein for the alpha IV monoclonal antibody suggests an alteration of
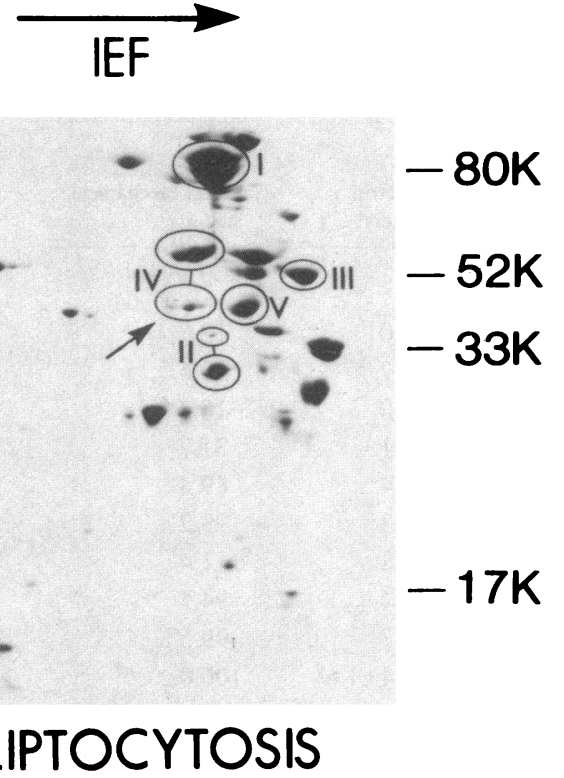

amide gradient). The five alpha spectrin domains are circled and indicated by Roman numerals. A decrease in the quantity of the smaller of two $\alpha$ IV fragments is indicated by the arrow.

the relevant epitope in the mutant's alpha IV domain. However, we do not know the location of this epitope within the alpha IV domain nor the nature of the mutation in alpha spectrin. Therefore, we cannot incriminate with certainty the alpha IV domain. Furthermore, it is unclear how a mutation in the alpha IV domain would interfere with spectrin dimer-dimer association, a function of the alpha I domain.

The striking difference in the amount of the mutant spectrin present in the various members of this kindred is a surprising finding in a disorder thought to be autosomal dominant. In three family members $\left(\mathrm{I}_{1}, \mathrm{III}_{1}\right.$, and $\left.\mathrm{III}_{2}\right), 40-45 \%$ of the alpha spectrin was mutant protein. This is near the $50 \%$ value expected for an autosomal dominant disorder and similar to that observed in two other spectrin defects in which the relative amount of mutant spectrin could be quantitated $(15,39)$. However, erythrocytes from the other three affected family members contained considerably less mutant protein (9.9-27.7\% of total alpha spectrin). This variability was not associated with a quantitative deficiency of total spectrin, as spectrin to band 3 ratios were normal. Thus, the variable percentage of mutant alpha spectrin reflects a variability in the actual quantity of the mutant protein among the affected individuals. Marchesi and her co-workers have recently described similar variability in a kindred with $\mathrm{HE}$ caused by another alpha-spectrin defect (14). The relative amount of the intact mutant spectrin was not quantitated, but these individuals showed a variable susceptibility of the alpha I tryptic domain to limited tryptic digestion, suggesting varying amounts of mutant spectrin.

It has been demonstrated in both avian (40) and mammalian (41) erythroid progenitors that alpha spectrin is synthesized in excess of beta spectrin. Because the membrane skeleton of mature erythrocytes contains an equal number of each of these complementary chains, it follows that many molecules of alpha spectrin are never incorporated into the membrane skeleton. Hence, variability in the relative amount of the mutant protein detected in circulating erythrocytes does not necessarily indicate 
equivalent variability in the translation of a mutant structural gene. For example, a decrease in the production of normal alpha spectrin on one chromosome might be undetectable in an otherwise normal RBC but would increase the relative amount of a mutant alpha spectrin inherited on the other chromosome (35). One difficulty with postulating a second (silent) inherited defect affecting spectrin synthesis is the need to invoke separate inheritance of such a defect in two generations (I and III, see Fig. 1). These two generations both exhibit greater amounts of the alpha mutant than is seen in the intervening generation (II). Furthermore, in generation II itself, there is variability in the amount of mutant spectrin. Clearly, in vitro studies of spectrin synthesis would be of considerable interest in this family.

We also considered the possibility that the variable quantity of the mutant protein was the result of posttranslational modification of the alpha spectrin. In a preliminary attempt to address this issue, we compared the quantity of alpha mutant in erythrocytes separated by buoyant density. No differences in the percentage of alpha spectrin mutant were observed in the comparison of these density (age) separated erythrocytes. However, because membrane skeleton assembly is essentially completed before extrusion of the nucleus, these data do not exclude the possibility of posttranslational modification of the alpha spectrin before its incorporation into the skeleton.

The structural gene for alpha spectrin is located on the $\mathrm{q}$ arm of human chromosome 1 (42) near the gene for the Fy blood group antigen. Because a gene dictating the posttranslational modification of alpha spectrin might reside on a different region of chromosome 1 or on another chromosome, we searched for linkage of the mutant alpha spectrin with the Fy antigen. Fy phenotyping of family members was inconclusive of linkage to the elliptocytosis defect. Hopefully, studies of genetic material from this family will reveal the precise nature and location of the mutation and help deliniate the mechanism(s) responsible for the different amounts of mutant protein found in affected family members.

\section{Acknowledgments}

We gratefully acknowledge E. Epstein, Jr. and J. Bonifas for helpful advice, P. Toy and M. Reid for the performance and interpretation of Fy blood group phenotyping, M. Rossi for technical assistance, J. Lee and V. Bible for editorial assistance, and the family described in this report for their interest and enthusiastic cooperation. The anti-spectrin antibodies were gifts from S. C. Liu, J. Palek, and J. Chasis.

This work was supported in part by funding from the National Institutes of Health (AM-32094, AM-26263). Dr. Lane is recipient of a National Research Service Award (HD 06633).

\section{References}

1. Lux, S. E. 1979. Spectrin-actin membrane skeleton of normal and abnormal red blood cells. Semin. Hematol. 16:21-51.

2. Marchesi, V. T. 1983. The red cell membrane skeleton: recent progress. Blood. 61:1-11.

3. Mohandas, N., J. A. Chasis, and S. B. Shohet. 1983. The influence of membrane skeleton on red cell deformability, membrane material properties, and shape. Semin. Hematol. 20:225-242.

4. Tchernia, G., N. Mohandas, and S. B. Shohet. 1981. Deficiency of skeletal membrane protein band 4.1 in homozygous hereditary elliptocytosis. Implications for erythrocyte membrane stability. J. Clin. Invest. 68:454-460.

5. Agre, P., E. P. Orringer, D. H. K. Chiu, and V. Bennett. 1981. A molecular defect in two families with hemolytic poikilocytic anemia.
Reduction of high affinity membrane binding sites for ankyrin. J. Clin. Invest. 68:1566-1576.

6. Coetzer, T., and S. S. Zail. 1981. Tryptic digestion of spectrin in variants of hereditary elliptocytosis. J. Clin. Invest. 67:1241-1248.

7. Liu, S.-C., J. Palek, and J. Prchal. 1981. Defective membrane skeleton assembly in hereditary elliptocytosis. In Erythrocyte Membranes 2: Recent Clinical and Experimental Advances. W. Kruckeberg, J. Eaton, and G. Breuer, editors. Alan R. Liss Inc., New York. 157-165.

8. Coetzer, T., and S. Zail. 1982. Spectrin tetramer-dimer equilibrium in hereditary elliptocytosis. Blood. 59:900-905.

9. Liu, S.-C., J. Palek, and J. Prchal. 1982. Defective spectrin dimerdimer association in hereditary elliptocytosis. Proc. Natl. Acad. Sci. USA 79:2072-2076.

10. Lawler, J., S.-C. Liu, J. Palek, and J. Prchal. 1984. A molecular defect of spectrin in a subset of patients with hereditary elliptocytosis. Alterations in the $\alpha$-subunit domain involved in spectrin self-association. J. Clin. Invest. 73:1688-1695.

11. Zail, S. S., and T. L. Coetzer. 1984. Defective binding of spectrin to ankyrin in a kindred with recessively inherited hereditary elliptocytosis. J. Clin. Invest. 74:753-762.

12. Lecomte, M.-C., D. Dhermy, C. Solis, A. Ester, C. Feo, H. Gautero, O. Bournier, and P. Boivin. 1985. A new abnormal variant of spectrin in black patients with hereditary elliptocytosis. Blood. 65:1208-1217.

13. Lawler, J., T. L. Coetzer, J. Palek, H. S. Jacob, and N. Luban. 1985. Sp alpha 1/65: a new variant of the alpha subunit of spectrin in hereditary elliptocytosis. Blood. 66:706-709.

14. Marchesi, S. L., W. J. Knowles, J. S. Morrow, M. Bologna, and V. T. Marchesi. 1986. Abnormal spectrin in hereditary elliptocytosis. Blood. 67:141-151.

15. Dhermy, D., M. C. Lecomte, M. Garbarz, O. Bournier, C. Galand, H. Gautero, C. Feo, N. Alloisio, J. Delaunay, and P. Boivin. 1982. Spectrin $\beta$-chain variant associated with hereditary elliptocytosis. J. Clin. Invest. 70:707-715.

16. Ohanian, V., J. P. Evans, and W. B. Gratzer. 1985. A case of elliptocytosis associated wtih a truncated spectrin chain. Brit. J. Haematol. 61:31-39.

17. Nielsen, J. A., and K. W. Strunk. 1968. Homozygous hereditary elliptocytosis as the cause of haemolytic anemia in infancy. Scand. $J$. Haematol. 5:486-496.

18. Mentzer, W. C., T. Turetsky, N. Mohandas, S. E. Schrier, C-S. C. Wu, and H. Koenig. 1984. Identification of the hereditary pyropoikilocytosis carrier state. Blood 63:1439-1446.

19. Clark, M. R., R. C. Unger, and S. B. Shohet. 1978. Monovalent cation composition and ATP and lipid content of irreversibly sickled cells. Blood. 51:1169-1178.

20. Beutler, E., and C. West. 1976. The removal of leukocytes and platelets from whole blood. J. Lab. Clin. Med. 88:328-333.

21. Dodge, J. T., C. Mitchell, and D. J. Hanahan. 1963. The preparation and chemical characteristics of hemoglobin free ghosts of human erythrocytes. Arch. Biochem. Biophys. 700:119-130.

22. Fairbanks, G., T. L. Steck, and D. F. H. Wallach. 1971. Electrophoretic analysis of the major polypeptides of the human erythrocyte membrane. Biochemistry. 10:2606-2617.

23. Laemmli, U. K. 1970. Cleavage of structural proteins during the assembly of the head of bacteriophage T4. Nature (Lond.). 227:680-685.

24. Fenner, C., R. R. Traut, D. T. Mason, and J. Wickman-Coffelt. 1975. Quantification of Coomassie blue stained proteins in polyacrylamide gels based on analyses of eluted dye. Anal. Biochem. 63:595-602.

25. Burnette, W. N. 1981. Western blotting: electrophoretic transfer of proteins from sodium-dodecyl sulfate-polyacrylamide gels to unmodified nitrocellulose and radiographic detection with antibody and radioiodinated protein A. Anal. Biochem. 112:195-203.

26. Epstein, E. H., Jr., and J. M. Bonifas. 1985. Recessive X-linked ichthyosis: lack of immunologically detectable steroid sulfatase enzyme protein. Hum. Genet. 71:201-205.

27. Wyatt, J. L., A. C. Greenquist, and S. B. Shohet. 1977. Analysis of phosphorylated tryptic peptides of spectrin from human erythrocyte membranes. Biochem. Biophys. Res. Commun. 79:1279-1285. 
28. Lawler, J., S.-C. Liu, J. Palek, and J. Prchal. 1982. Molecular defect of spectrin in hereditary pyropoikilocytosis. Alterations in the trypsin-resistant domain involved in spectrin self-association. J. Clin. Invest. 70:1019-1030.

29. Liu, S.-C., G. Fairbanks, and J. Palek. 1977. Spontaneous, reversible protein cross-linking in the human erythrocyte membrane: temperature and $\mathrm{pH}$ dependence. Biochemistry 16:4066-4074.

30. Knowles, W. J., J. S. Morrow, D. W. Speicher, H. S. Zarkowsky, N. Mohandas, W. C. Mentzer, S. B. Shohet, and V. T. Marchesi. 1983. Molecular and functional changes in spectrin from patients with hereditary pyropoikilocytosis. J. Clin. Invest. 71:1867-1877.

31. Knowles, W. J., and M. Bologna. 1983. Isolation of the chemical domains of human erythrocyte spectrin. Methods Enzymol. 96:305-313.

32. O'Farrell, P. H. 1975. High resolution two-dimensional electrophoresis of proteins. J. Biol. Chem. 250:4007-4021.

33. Speicher, D. W., J. S. Morrow, W. J. Knowles, and V. T. Marchesi. 1982. A structural model of human erythrocyte spectrin. J. Biol. Chem. 257:9093-9101.

34. Mohandas, N., M. R. Clark, B. P. Health, M. Rossi, L. C. Wolfe, S. E. Lux, and S. B. Shohet. 1982. A technique to detect reduced mechanical stability of red cell membranes: relevance to elliptocytic disorders. Blood. 59:768-774.

35. Palek, J. 1985. Hereditary elliptocytosis and related disorders. Clinics Haematol. 14:45-87.

36. deJong, W. W., A. Zweers, and L. H. Cohen. 1978. Influence of single amino acid substitutions on electrophoretic mobility of sodium dodecyl sulfate-protein complexes. Biochem. Biophys. Res. Commun. 82:532-539.

37. Noel, D., K. Nikaido, and G. F.-L. Ames, 1979. A single amino acid substitution in a histidine-transport protein drastically alters its mobility in sodium dodecyl sulfate-polyacrylamide gel electrophoresis. Biochemistry 18:4159-4165.

38. Vandekerckhove, J., J. Leavitt, T. Kakunaga, and K. Weber. 1980. Coexpression of a mutant beta actin and the two normal beta and gamma cytoplasmic actins in a stably transformed human cell line. Cell 22:893-899.

39. Wolfe, L. C., K. M. John, J. C. Falcone, A. M. Byrne, and S. E. Lux. 1982. A genetic defect in the binding of protein 4.1 to spectrin in a kindred with hereditary spherocytosis. N. Engl. J. Med. 307:13671374.

40. Blikstad, I., W. J. Nelson, R. T. Moon, and E. Lazarides. 1983. Synthesis and assembly of spectrin during avian erythropoiesis: stoichiometric assembly but unequal synthesis of alpha and beta spectrin. Cell 32:1081-1091.

41. Hanspal, M., and J. Palek. 1985. Synthesis and assembly of spectrin in mammalian erythroid cells: alpha spectrin is synthesized in excess of beta spectrin. Blood. 66(Suppl. 1):33a. (Abstr.)

42. Huebner, K., A. P. Palumbo, M. Isobe, C. A. Kozak, S. Monaco, G. Rovera, C. M. Croce, and P. J. Curtis. 1985. The alpha spectrin gene is on chromosome 1 in the mouse and man. Proc. Natl. Acad. Sci. USA. $82: 3790-3793$. 